\title{
Rewiring the Lesioned Brain: Electrical Stimulation for Post-Stroke Motor Restoration
}

\author{
Shi-chun Bao, ${ }^{\mathrm{a}}$ Ahsan Khan, ${ }^{\mathrm{a}}$ Rong Song, ${ }^{\mathrm{b}}$ Raymond Kai-yu Tong ${ }^{\mathrm{a}}$ \\ ${ }^{a}$ Department of Biomedical Engineering, The Chinese University of Hong Kong, Hong Kong, China

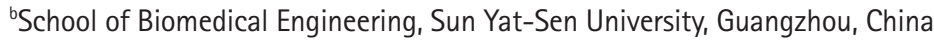

Electrical stimulation has been extensively applied in post-stroke motor restoration, but its treatment mechanisms are not fully understood. Stimulation of neuromotor control system at multiple levels manipulates the corresponding neuronal circuits and results in neuroplasticity changes of stroke survivors. This rewires the lesioned brain and advances functional improvement. This review addresses the therapeutic mechanisms of different stimulation modalities, such as noninvasive brain stimulation, peripheral electrical stimulation, and other emerging techniques. The existing applications, the latest progress, and future directions are discussed. The use of electrical stimulation to facilitate post-stroke motor recovery presents great opportunities in terms of targeted intervention and easy applicability. Further technical improvements and clinical studies are required to reveal the neuromodulatory mechanisms and to enhance rehabilitation therapy efficiency in stroke survivors and people with other movement disorders.

\author{
Correspondence: Raymond Kai-yu Tong \\ Department of Biomedical \\ Engineering, The Chinese University \\ of Hong Kong, Office Rm 1120A, \\ William M.W. Mong Engineering \\ Building, Shatin, N.T., Hong Kong, \\ China \\ Tel: +852-3943-8454 \\ Fax: +852-2603-5558 \\ E-mail:kytong@cuhk.edu.hk
}

Received: November 5, 2019

Revised: January 3, 2020

Accepted: January 6, 2020

Keywords Electric stimulation; Stroke; Motor recovery; Transcranial direct current stimulation; Transcranial magnetic stimulation; Neuromuscular electrical stimulation

\section{Introduction}

Stroke is the second leading cause of death and the leading cause of disability worldwide, recent study showed that its disability-adjusted life year is nearly 113 million globally. ${ }^{1}$ Stroke incidence and mortality increases with age, and for the coming aging population, more stroke cases are expected which would induce a severe burden on the society. ${ }^{2}$ About $20 \%$ of stroke patients die, whereas $80 \%$ of stroke survivors experience motor impairments contralateral to the lesioned hemisphere. ${ }^{3}$ Typical stroke symptoms include unilateral motor weakness, limb hemiparesis, spasticity, gait disturbance, and loss of coordination. ${ }^{4}$ More than half of stroke patients cannot fully recover from motor impairments, and the quality of their life is substantially affected. ${ }^{5}$

Motor control is the ability to regulate mechanisms requisite to locomotion. ${ }^{6}$ The hierarchical motor control process involves multiple brain structures, as illustrated in Figure 1. Both the central nervous system (including the cerebral cortex, cerebellum, brain stem, and spinal cord) and peripheral extremities are involved in the motor control process. ${ }^{7,8}$ Corticospinal tract (CST) derives from the sensorimotor cortex, projecting its output to spinal interneuron or motoneuron circuits. It is essentially the dominant descending pathway for the motor control process in primates. ${ }^{9}$ The proprioceptor and other sensory inputs may transmit back to the sensorimotor cortex through the spinal tracts. ${ }^{10}$ Motor commands are transferred from the cortex to the reticular formation in the brainstem, and further transmitted to spinal interneuron or motoneuron circuits and peripheral extremities via the reticulospinal tract. ${ }^{11}$ The reticulospinal tract is crucial for human locomotion, balance, and coordination. Integrity of the motor control system is pivotal 


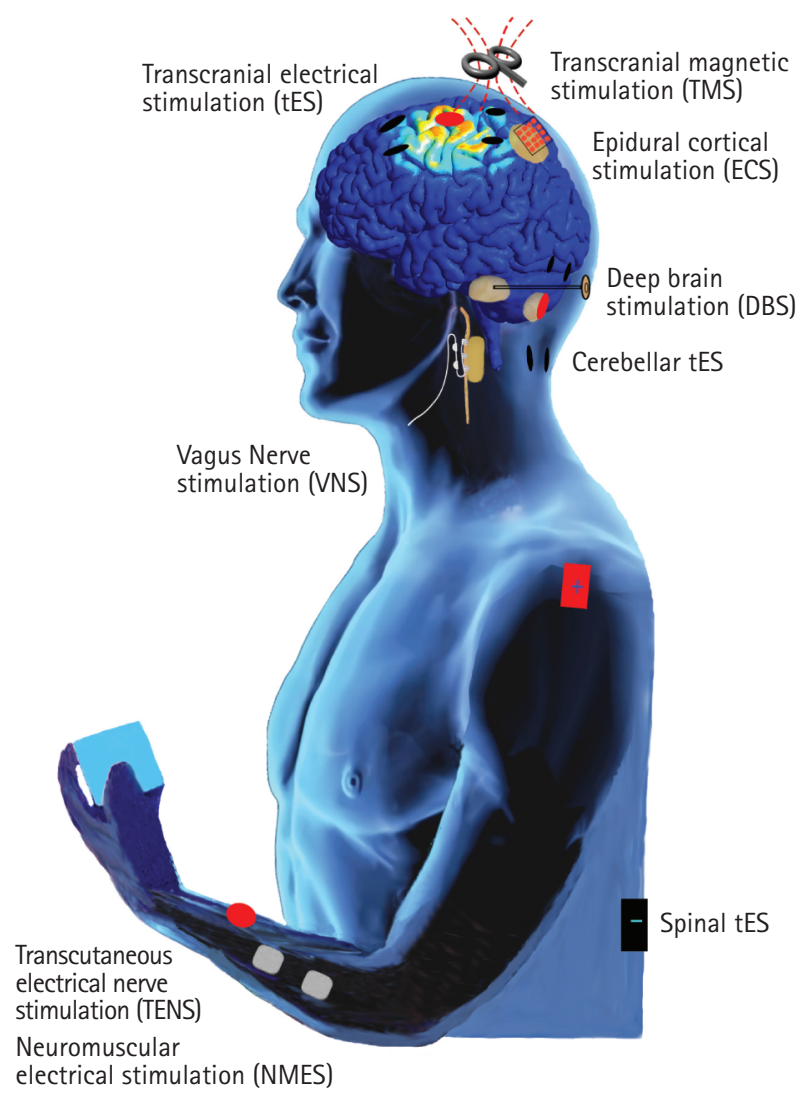

Figure 1. Typical electrical stimulation modalities for post-stroke motor restoration. Finite element modeling result of transcranial electrical stimulation (tES)-induced electrical field is illustrated.

for human locomotion. Therefore, damage to sensorimotor or higher-order brain regions results in motor impairment. ${ }^{12}$ In such a case, rehabilitation is essential in regaining functional improvement or restoration. Post-stroke motor restoration is challenging due to genetic, pathophysiologic, sociodemographic, and other clinical factors. ${ }^{13}$ Hence, manipulating related neural circuits and rewiring the lesioned brain might be critical factors for post-stroke motor recovery.

In 1949, neuropsychologist Donald Hebb ${ }^{14}$ proposed the rule that "Neurons that fire together, wire together." Hebb's rule provided the theoretical foundations that homosynaptic and heterosynaptic activities facilitate synaptic formation and consolidation during motor rehabilitation. ${ }^{14}$ Neuroplasticity could be augmented through rehabilitation strategies, ${ }^{15,16}$ such as basic task-oriented training (TOT). However, TOT alone cannot effectively alleviate motor impairment and restore motor functions. ${ }^{17}$ Unlike pharmacological therapy, electrical stimulation provides a more targeted intervention to damaged motor neural circuits, resulting in better functional recovery for patients with motor impairments. ${ }^{18,19}$

Various electrical stimulation modalities have been used to promote neuroplasticity and facilitate post-stroke motor recovery in different levels of the neuromotor control system. Tentative stimulation targets include the motor cortex, peripheral extremities, cerebellum, deep brain, vagus nerve, and other related areas, as shown in Figure 1. Table 1 summarizes the milestones of various electrical stimulation modalities in poststroke motor recovery, with classification, main findings, and references listed in a chronological order. Among these protocols, noninvasive brain stimulation (NIBS) and peripheral electrical stimulation protocols have been extensively employed. Other related stimulation protocols are still in the prefatory laboratory or preclinical exploration stages. Figure 2 presents an intuitive summary to Table 1, highlighting the timeline and a brief history of typical electrical stimulation techniques in post-stroke motor recovery. Although neural electrical stimulation was proposed nearly 60 years ago, its application in poststroke motor recovery was not actualized until the end of the twentieth century. Markedly, the past 5 years witnessed progressive developments in electrical stimulation techniques and availability of new interventions in post-stroke motor recovery. Here, we provide a detailed review of multi-level electrical stimulation-based post-stroke motor recovery summarizing the published studies and future trends in this field.

\section{Noninvasive brain stimulation}

NIBS has been utilized as a stand-alone or supplementary rehabilitation tool in stroke related motor recovery. ${ }^{19}$ NIBS modulates neural synaptic plasticity and motor skill acquisition beyond the stimulation period. Such modulatory effects facilitate motor learning and neurorehabilitation process, and further enhance paretic limb motor function. ${ }^{20}$ NIBS modalities reviewed here include transcranial electrical stimulation (tES) or transcranial magnetic stimulation (TMS), TMS is also included as it induced electric currents through electromagnetic induction. Cerebellum and spinal cord stimulation protocols are also discussed.

\section{Transcranial electrical stimulation}

As a representative NIBS protocol, tES modulates cortical excitability and induces CST changes lasting beyond stimulation periods. ${ }^{21-23}$ Pioneer tES applications date back 2,000 years ago during the Greco-Roman period. Electricity from organs of electric fish was used to treat pain, limb paresis, and other symptoms. ${ }^{24}$ Earlier studies with rat models demonstrated neuronal depolarization after anode electrical stimulation. ${ }^{25} \mathrm{Mod}-$ ern noninvasive transcranial direct current stimulation (tDCS) studies began at the end of the 20th century. ${ }^{26,27}$

Noninvasive tES is powered by battery-based electrical cir- 
Table 1. Milestones of various electrical stimulation modalities in post-stroke motor recovery

\begin{tabular}{|c|c|c|}
\hline \multirow{2}{*}{ Stimulation modalities } & \multicolumn{2}{|l|}{ Representative studies } \\
\hline & Key findings & Reference \\
\hline \multicolumn{3}{|l|}{ Noninvasive brain stimulation } \\
\hline \multirow[t]{8}{*}{$\mathrm{tES}$} & ES induced neuronal depolarization in rats & Bindman et al. $(1962)^{25}$ \\
\hline & $\begin{array}{l}\text { Conventional tDCS modulation effects of motor excitability in healthy } \\
\text { subjects }\end{array}$ & $\begin{array}{l}\text { Priori et al. }(1998)^{26}, \\
\text { Nitsche et al. }(2000)^{27}\end{array}$ \\
\hline & tDCS facilitates post-stroke motor recovery & Hummel et al. $(2005)^{49}$ \\
\hline & High definition tDCS with increase focality & Borckardt et al. $(2012)^{28}$ \\
\hline & Network-based tDCS targeting multiple-area & Fischer et al. $(2017)^{57}$ \\
\hline & Online closed-loop EEG-tDCS & Leite et al. $(2017)^{59}$ \\
\hline & In vivo neuronal circuits modulated by tDCS for human and rats & Vöröslakos et al. (2018) ${ }^{44}$ \\
\hline & Gait-synchronized tACS & Koganemaru et al. (2019) ${ }^{61}$ \\
\hline \multirow[t]{7}{*}{ TMS } & TMS influence on healthy motor cortex & Barker et al. $(1985)^{63}$ \\
\hline & rTMS cortical excitability effects in healthy & Maeda et al. $(2000)^{67}$ \\
\hline & rTMS in post-stroke motor recovery & Takeuchi et al. $(2005)^{68}$ \\
\hline & PAS increase MEP in healthy subjects & Fratello et al. $(2006)^{74}$ \\
\hline & TBS applications in healthy subjects & Huang et al. $(2007)^{72}$ \\
\hline & Unknown rTMS parameters in stroke, review & Hao et al. $(2013)^{76}$ \\
\hline & Multi-locus TMS to increase targeting & Koponen et al. $(2018)^{82}$ \\
\hline \multirow[t]{5}{*}{ Cerebellar and spinal cord stimulation } & Cerebellar tDCS influence CBI in healthy & Galea et al. (2009) 88 \\
\hline & Cerebellar tDCS to improve motor skill learning and adaptation in healthy & $\begin{array}{l}\text { Doppelmayr et al. }(2016)^{90} \\
\text { Erfmann }(2018)^{89}\end{array}$ \\
\hline & Cerebellar tACS and stroke neuroplasticity & Naro et al. $(2016)^{93}$ \\
\hline & Cerebellar tDCS in stroke standing balance & Zandvliet et al. $(2018)^{91},(2019)^{92}$ \\
\hline & $\begin{array}{l}\text { Combined effect of spinal tDCS, robot training, and cerebellar/cortical } \\
\text { tDCS }\end{array}$ & $\begin{array}{l}\text { Picelli et al. }(2015)^{97},(2018)^{98} \\
(2019)^{99}\end{array}$ \\
\hline \multicolumn{3}{|l|}{ Peripheral electrical stimulation } \\
\hline \multirow[t]{6}{*}{ NMES } & FES in post-stroke hemiplegic gaiting & Liberson et al. $(1961)^{105}$ \\
\hline & Implanted NMES system & Peckham et al. (1988) ${ }^{106}$ \\
\hline & Myoelectric control of NMES & Cauraugh et al. (2000) $)^{110}$ \\
\hline & $\mathrm{BCl}$ control of NMES & Meng et al. (2008) $)^{109}$ \\
\hline & Invasive $\mathrm{BCl}-\mathrm{NMES}$ with fine movement & Bouton et al. (2016) $)^{119}$ \\
\hline & High density NMES to allow fine control & Annetta et al. (2019) 20 \\
\hline \multirow[t]{3}{*}{ TENS } & TENS for pain relief & Augustinsson et al. (1977) 122 \\
\hline & TENS for stroke sensorimotor functions & Peurala et al. (2002) $)^{124}$ \\
\hline & TENS in post-stroke motor recovery, review & Grant et al. (2018) 129 \\
\hline \multicolumn{3}{|l|}{ Emerging electrical stimulation techniques } \\
\hline \multirow[t]{4}{*}{ DBS } & DBS in limb paresis after stroke & Phillips et al. $(2000)^{138}$ \\
\hline & DBS for DTC pathway in stroke & Machado et al. (2012) ${ }^{143}$ \\
\hline & Noninvasive interference DBS & Grossman et al. $(2017)^{145}$ \\
\hline & Cerebellar DBS-based post-stroke motor recovery, review & Wathen et al. (2018) $)^{144}$ \\
\hline \multirow[t]{3}{*}{ ECS } & ECS in rat stroke model & Brown et al. (2006) $)^{147}$ \\
\hline & Phase I, II clinical trials in stroke patients & Levy et al. (2008) $)^{149},(2016)^{150}$ \\
\hline & Array focal cortical stimulation & Yang et al. (2017) ${ }^{155}$ \\
\hline
\end{tabular}


Table 1. Continued

\begin{tabular}{lll}
\hline \multirow{2}{*}{ Stimulation modalities } & \multicolumn{2}{c}{ Representative studies } \\
\cline { 2 - 3 } & \multicolumn{1}{c}{ Key findings } & \multicolumn{1}{c}{ Reference } \\
\hline VNS & Invasive VNS in stroke rat model & Khodaparast et al. (2013) $^{164}$ \\
& Noninvasive VNS in stroke rat model & Ay et al. (2016) ${ }^{167}$
\end{tabular}

tES, transcranial electrical stimulation; ES, electrical stimulation; $\mathrm{DDCS}$, transcranial direct current stimulation; EEG, electroencephalogram; tACS, transcranial alternating current stimulation; TMS, transcranial magnetic stimulation; rTMS, repetitive TMS; PAS, paired associative stimulation; MEP, motor evoked potential; TBS, theta burst stimulation; CBI, cerebellar brain inhibition; NMES, neuromuscular electrical stimulation; FES, functional electrical stimulation; $\mathrm{BCl}$, brain computer interface; TENS, transcutaneous electrical nerve stimulation; DBS, deep brain stimulation; DTC, dentatothalamocortical; ECS, epidural cortical stimulation; VNS, vagus nerve stimulation.

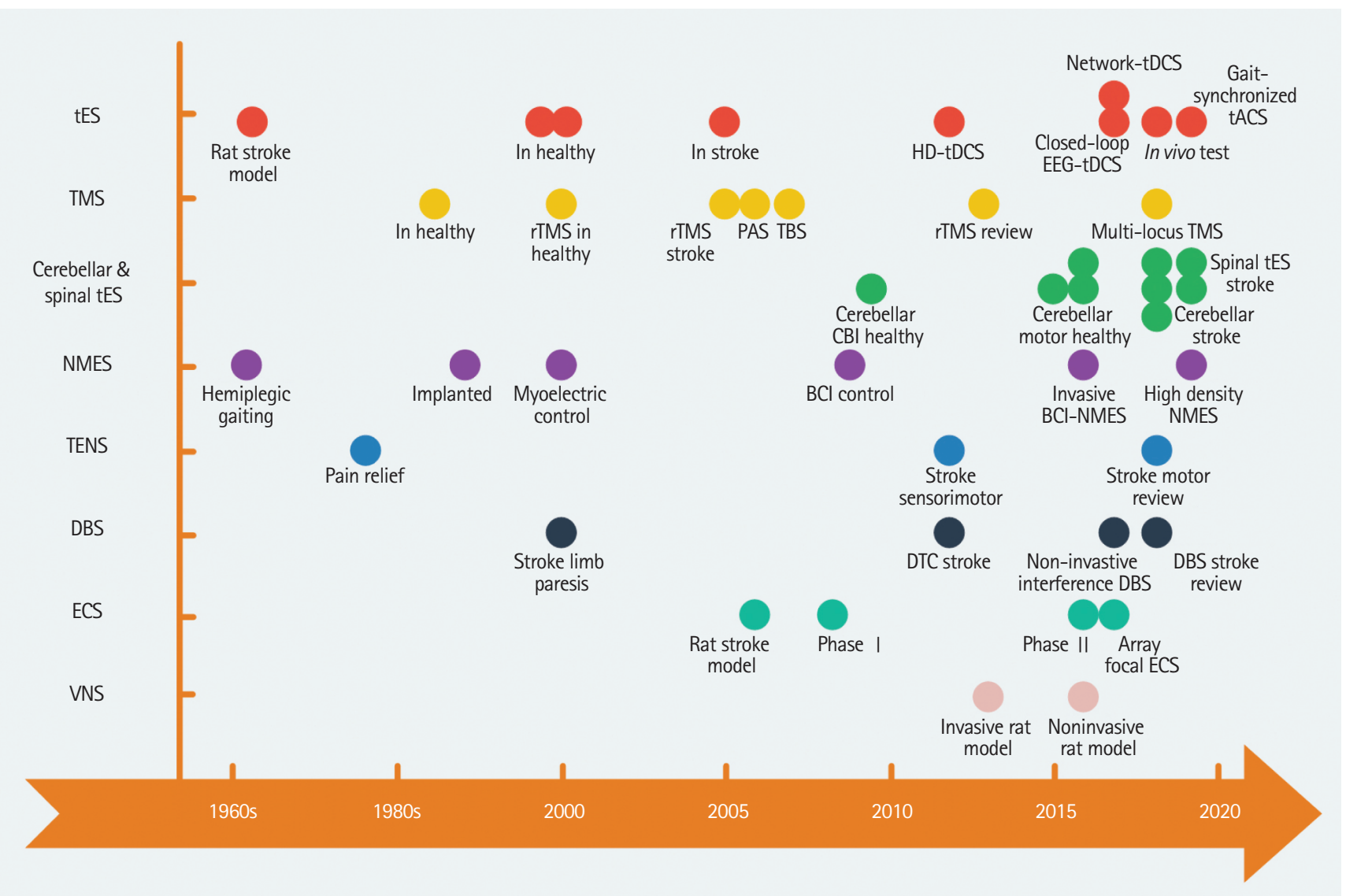

Figure 2. Timeline and brief history of representative electrical stimulation techniques in post-stroke motor recovery. Each dot represents one typical finding as shown in Table 1, different color indicates different stimulation modality. x-axis, year in sequence, before 2000, each tick means 20 years, after 2000 , each tick means 5 years; $y$-axis, different electrical stimulation techniques. tES, transcranial electrical stimulation; TMS, transcranial magnetic stimulation; NMES, neuromuscular electrical stimulation; TENS, transcutaneous electrical nerve stimulation; DBS, deep brain stimulation; ECS, epidural cortical stimulation; VNS, vagus nerve stimulation; tDCS, transcranial direct current stimulation; tACS, transcranial alternating current stimulation; EEG, electroencephalogram; rTMS, repetitive TMS; PAS, paired associative stimulation; TBS, theta burst stimulation; CBI, cerebellar brain inhibition; $\mathrm{BCl}$, brain computer interface; DTC, dentatothalamocortical.

cuits, the generated low-amplitude currents penetrate the skull and influence the brain area underneath stimulation sites. tES modifies transmembrane neuronal potential and further modulates cortical excitability. Essentially, tES with different parameter settings induces different modulation effects. ${ }^{24} \mathrm{~A}$ conventional tES system comprises a conductive rubber pad-based tES ( $5 \times 7 \mathrm{~cm}$, for example), while the newer high definition
(HD) tES with small ring-based electrodes has better focality and outperforms the conventional settings. ${ }^{28,29}$ Typical tES comprises tDCS and transcranial alternating current stimulation (tACS). tACS utilizes sinusoidal current with different stimulation frequencies and evokes cortical activations. Studies have reported that different stimulation frequencies lead to different modulatory effects. For instance, $10 \mathrm{~Hz}$ tACS enhanc- 
es motor learning significantly, ${ }_{1}^{30} 20 \mathrm{~Hz}$ tACS decreases beta band cortico-muscular coupling in finger tapping tasks, ${ }^{31}$ while 1 to $5 \mathrm{kHz}$ range tACS increases motor cortex excitability. ${ }^{32}$ Further studies are needed to translate tACS into clinical applications. Other versions of tES including transcranial random noise stimulation and transcranial pulsed current stimulation are not commonly used in stroke rehabilitation yet.

As the most frequently used tES modality, tDCS employs weak direct electrical current stimulation (around 0.5 to $2 \mathrm{~mA}$ ) with two or more electrodes placed on the primary motor cortex (M1) or its neighboring area for post-stroke motor recovery. Modulation after-effects of 10 to 20 minutes stimulation could last for about 30 to 40 minutes depending on the stimulation settings. ${ }^{24}$ Such stimulation induces polarity-dependent neural modulatory effects. Anode and cathode stimulation enhances and inhibits motor excitability, respectively. ${ }^{33}$ Particularly, tDCS induced persistent bidirectional modification of post-synaptic connections is similar to long-term potentiation (LTP, anode) and long-term depression (LTD, cathode). ${ }^{24}$ At the neuron level, tDCS generates glutamatergic plasticity with a modulatory effect on neurotransmitters and ion channels, including N-methyl-D-aspartate glutamate, and brain-derived neurotrophic factor (BDNF).

Pioneer study found that tDCS induced motor evoked potentials (MEPs) changes with TMS, which allows for reproducible measurement of cortical excitability. ${ }^{27}$ Other electrophysiological, hemodynamic, and neurophysiological measurement tools including functional magnetic resonance imaging (MRI), ${ }^{34}$ and functional near-infrared spectroscopy (fNIRS) ${ }^{35}$ have been utilized to scrutinize modulatory effects of tDCS. Additionally, the immediate modulation effects of tDCS on task-specific brain oscillation have been explored using electroencephalogram (EEG), electromyogram (EMG), and local field potential. ${ }^{36-38}$ These studies reported that tDCS modulates motor control process and induces cortical excitability changes. Moreover, tDCS fosters external limb properties of leg tibialis anterior muscle pinching, voluntary paretic ankle control, and isometric contraction myoelectric control. ${ }^{39-41}$ In addition to local modulatory effects underneath the stimulation area, tDCS also modulates regions distant from stimulation sites by influencing motor-related neural synchrony, including cortical connectivity, corticospinal excitability, and cortico-muscular coupling. For instance, anode $\mathrm{LDCS}$ over left M1 facilitated cortical synchronization in the alpha and lower bands of the frontal and parieto-occipital cortex, the high gamma frequency bands of the motor cortex, ${ }_{1}^{42}$ and increased functional coupling of EEG rhythms in the sensorimotor cortex. ${ }^{43}$ In vivo intracellular and extracellular measurements illustrated that neuronal circuits are instantaneously influenced by electrical stimulation in rats and human cadaver brains in situ. ${ }^{44}$ An recent study reported that anode HD-tDCS could promote cortico-muscular coherence in chronic stroke subjects, ${ }^{45}$ suggesting an enhanced cortico-muscular communication after HD-tDCS. Using diffusion MRI, tDCS strengthens the descending corticospinal pathway from M1 to target muscles during brain computer interface (BCI)-based stroke rehabilitation with increased CST integrity. ${ }^{46}$ In addition to modulating functional plasticity, recent evidence suggested that tDCS induces structural plasticity and physiological BDNF expressions. ${ }^{47}$

Post-stroke motor recovery relies on neuroplasticity and brain reorganization. Such reorganization appears in the ipsilesional motor cortex, contralesional area, or deep brain regions. ${ }^{48}$ tDCS was first applied in post-stroke motor recovery in $2005^{49}$ and has thereafter been extensively utilized. ${ }^{22,50,51}$ These bench-to-bedside studies have provided evidences that tDCS and task-specific motor training contributed to long-term post-stroke motor learning and recovery. Stroke focal lesion disrupts the balanced interhemispheric inhibition, with the over-inhibition of ipsilesional hemisphere preventing paretic limbs from acquiring better recovery. ${ }^{52}$ However, such interhemispheric competition models are still under scrutiny, a bimodal balance-recovery model was proposed for guiding neurorehabilitation in $2014 .^{53}$ tDCS is currently employed in either inhibiting the contralesional hemisphere or exciting the lesioned hemisphere, simultaneous stimulation of bilateral hemispheres has also been attempted. ${ }^{54}$ Such tDCS induced neuroplasticity could induce a long-lasting motor enhancement and recovery. Although several clinical experiments assessing the functional role of tDCS in post-stroke motor recovery have concluded with promising results, no consensus has been reached on its therapeutic efficacy from randomized controlled trials. $^{22,23,55}$ Intra-subject and inter-subject variability of response might limit the wide application of tDCS in stroke subjects. Large-scale, well-designed Phase III clinical trials and indepth understanding of tDCS modulatory mechanisms will enhance tDCS-based rehabilitation efficiency. In addition, biophysical models could predict treatment efficacy, elucidating the underlying mechanisms in different levels of post-stroke recovery. Several preliminary theoretical frameworks have been proposed towards understanding the effects of tES on neurorehabilitation. ${ }^{56}$ Future studies should provide more personalized and reliable models of tDCS-based neurorehabilitation.

tES electrode placement and the corresponding electric fields could also influence modulation results. Recent advancements in multichannel network-based tDCS showed better modulatory effects as compared to 2 to 5 channel tDCS in healthy subjects, indicating better tools for future stroke rehabilitation studies. ${ }^{57}$ In addition, it is imperative to employ computational 
modeling in obtaining optimized stimulation settings for individual stroke subjects, factoring in the impact of stroke lesion and the heterogeneity of brain anatomy. ${ }^{58} \mathrm{~A}$ recent closed-loop EEG-tDCS system introduced online control of electrical stimulation with promising clinical applications. ${ }^{59}$ To enable simultaneous stimulation and artifact-free recordings, advanced artifact removal strategies are required. ${ }^{60}$ Moreover, a recent pilot study reported that gait-synchronized tACS could facilitate gait recovery in stroke patients. ${ }^{61}$ When developing and advancing these state-of-art techniques, it will be vital to evaluate their reliability with large sample size randomized controlled clinical trials. Such systems will provide a blueprint on future rehabilitation applications.

\section{Transcranial magnetic stimulation}

TMS induces a transient time-varying magnetic field perpendicular to the stimulation coil, which further produces electric currents parallel to the coil underneath the cortical tissues. Electromagnetic induction results in focused electrical currents, further inducing neuronal depolarization and propagation of action potentials. ${ }^{62}$ Barker et al. ${ }^{63}$ introduced TMS as a potential neuromodulation tool on the human motor cortex, TMS has since then been utilized for either physiological measurement or neuromodulation depending on stimulation settings. ${ }^{64}$ Single-pulse and paired-pulse TMS could measure the neurophysiological properties like MEPs and intracortical excitability. ${ }^{65,66}$ Repetitive TMS (rTMS) and patterned TMS modulate cortical excitability beyond stimulation period depending on stimulation settings. ${ }^{52,67}$ High-frequency rTMS (usually $\geq 5 \mathrm{~Hz}$ ) excites the brain, while low-frequency rTMS $(\leq 1 \mathrm{~Hz})$ inhibits cortical excitability. Modulation of stroke related neural circuitry and cortical substrates implies potential applications of rTMS in post-stroke rehabilitation.

Several studies have investigated the functional role of rTMS in motor recovery in stroke subjects. ${ }^{68,69}$ Like tES, rTMS influences neuroplasticity from synaptic connections similar to LTP and LTD process. It modulates the imbalanced interhemispheric inhibition between hemispheres, by either inhibiting the contralesional hemisphere or exciting the lesioned hemisphere. ${ }^{54}$ Excitatory rTMS could facilitate synchronicity of neural firing of ipsilesional cortical regions and further harness neuroplasticity following a stroke. Additional corticospinal pathways could also be activated and adjacent lesion areas could be recruited. ${ }^{70}$ Due to potential risk of rTMS-induced seizure in stroke patients, ${ }^{71}$ it is necessary to follow a strict screening process before conducting rTMS-based clinical trials. Simple rTMS induces modulatory effects for a few minutes, while theta burst stimulation (TBS) with subthreshold high-frequency stimula- tion (for example, $50 \mathrm{~Hz}$ ) induce modulation for about 30 to 60 minutes, the intermittent TBS promotes while continuous pattern inhibits cortical activity, respectively. ${ }^{72}$ When magnetic stimulus on the contralateral M1 was paired with peripheral nerve stimulus, it presented as a potential therapeutic intervention tool for post-stroke recovery. ${ }^{73}$ For such paired associative stimulation (PAS), M1 corticospinal excitability was modulated by the repeated pairing of the two stimuli, and the modulatory effects were linked to the interstimulus interval. ${ }^{74}$

Though numerous rTMS-based clinical trials have been conducted, there is no consensus on the adjunct therapeutic effects of rTMS. Therefore, the clinical applications of rTMS in post-stroke motor recovery are limited. ${ }^{75,76}$ Moreover, randomized controlled trials on stroke subjects were still lacking, and adjuvant use of rTMS with constraint-induced therapy showed no significant enhancement in an exploratory randomized clinical trial. ${ }^{77}$ Optimal protocols and stimulation parameter settings differ across subjects. ${ }^{78}$ Randomized controlled clinical trials with large sample size are required to determine the long-term and therapeutic effects of rTMS. Combination of rTMS with other intervention techniques could enhance poststroke motor recovery. Nevertheless, many neurophysiological processes following a stroke are involved in rTMS-based rehabilitation. In addition, the underlying mechanisms of rTMS neural circuit modulation remain only partially understood, greatly limiting the wide application of rTMS in post-stroke motor recovery. Computational modeling could be valuable in providing insights on fundamental cause and effect principles. Cortical networks and corticospinal changes following rTMS could be investigated by multimodal neurophysiological measures in animal models and a wide variety of stroke patients. ${ }^{79-81}$ Recent advancements in multi-locus TMS could contribute to individualized multiple-region stimulation therapy and further enhance neuroplasticity. ${ }^{82}$

\section{Cerebellar and spinal cord stimulation}

The cerebellum is a vital structure in movement control and coordination, including balance maintenance, gait, and fine motor skills. ${ }^{83}$ It is connected to $\mathrm{M} 1$, premotor, prefrontal, and other cerebral regions. The cerebellum is an essential part of error-based motor learning process, and the LTD-like plasticity of Purkinje cells in the cerebellum is associated with Hebbian learning. ${ }^{84}$ Cerebellar activities depend on the descending inputs from the contralateral cerebrum, and the ascending inputs from the cerebellum provide feedback to M1. Therefore, the cerebellum is involved in synchronization of both sensory input and motor output. ${ }^{85}$ Additionally, cerebellar excitability is correlated to motor adaptation in healthy and stroke subjects, im- 
plying that its neuroplasticity in sensorimotor learning could boost motor recovery. ${ }^{86}$ Not all stroke subjects can acquire motor recovery with noninvasive cortical stimulation. Alternatively, cerebellar tES shows promise in motor rehabilitation in stroke patients with a lesion in the cerebellum and other related regions. ${ }^{87}$

Pioneer cerebellar tDCS study investigated polarity-dependent modulation effects of cerebello-brain connectivity (cerebellar brain inhibition [CBI]) on healthy subjects, both anode and cathode stimulation protocols were effective in changing motor performance. Cathode stimulation results in decreased CBI by enhanced LTD of Purkinje cells, but did not induce M1 or corticospinal changes.$^{88} \mathrm{~A}$ single cerebellar tDCS training session for swallowing skill was sufficient to improve swallowing performance in healthy subjects, but it was not enough for stroke patients with dysphagia. ${ }^{89}$ Furthermore, cerebellar HDtDCS facilitated motor adaptation in healthy subjects, whereas HD-tDCS on M1 could not have such effects. ${ }^{90}$ In another proof-of-concept study, short-term contralesional cerebellar tDCS promoted standing balance performance in chronic stroke patients. ${ }^{91,92}$ Nevertheless, randomized controlled trials with a larger sample size are necessary to resolve inter-individual differences in the therapeutic interventions. To achieve qualitative functional improvements, optimal timing and dosage should also be determined. Further studies employing neuroimaging techniques are necessary to unravel the underlying neuromodulation effects following a stroke. In a study by Naro et al., ${ }^{93}$ different cerebellar tACS protocols resulted in different $\mathrm{CBI}$-sustaining Purkinje cell responses, affecting neuroplasticity of specific cerebellar pathways. Although several studies have been conducted, therapeutic applications of cerebellar stimulation are still in preliminary stages. Future studies should investigate the functional role of cerebellar stimulation in the corticospinal and corticobulbar motor control process. Moreover, cerebellar stimulation electrical flow, its corresponding modulatory effects and long-term impacts should be thoroughly evaluated.

The spinal cord contains neuronal circuits, mediating locomotion activities and segmental spinal reflexes. It is a bidirectional integration center for descending motor and ascending sensory feedback signals. ${ }^{94}$ Unlike tES, investigation on spinal cord stimulation in post-stroke motor recovery began in the recent decade. Spinal cord stimulation can modulate both the local and distal neural circuits, and induce neurophysiological and behavioral changes. ${ }^{95,96}$ To investigate the combined effects of transcutaneous spinal direct current stimulation (tsDCS) on cortical tDCS or cerebellar tDCS, Picelli et al. ${ }^{97-99}$ conducted several double-blinded, randomized controlled gait training clinical trials.
Anode tDCS combined with cathode tsDCS enhanced the effect of robot-assisted gait training (RAGT) in chronic stroke patients, larger enhancement in gait cadence was identified with anode tDCS+cathode thoracic tsDCS as compared to after tDCS or tsDCS alone. ${ }^{97}$ Similarly, cathode cerebellar tDCS+tsDCS+RAGT resulted in higher improvements in walking capacity and gait cadence in chronic ischemic stroke and supratentorial stroke patients. ${ }^{98,99}$ Though several clinical trials have been conducted, the rationale of tsDCS in stroke patients and the mechanism of spinal locomotion control remain unclear. Future studies should elucidate the mechanism of tsDCS modulation effects on the local spinal, supra-spinal, and intracortical motor control process. tsDCS could provide a potential therapeutic tool in various movement disorders.

\section{Peripheral electrical stimulation}

Peripheral electrical stimulation has been investigated for more than half of a century to activate bladder voiding, to relieve pelvic pain and other symptoms. Neuromuscular electrical stimulation (NMES) has mainly two forms in motor rehabilitation after stroke, particularly, functional electrical stimulation (FES) has been used to facilitate voluntary movement, while therapeutic electrical stimulation was used for strengthening muscle, reducing spasticity, and inducing motor recovery in paralyzed stroke patients. ${ }^{13,19}$ Transcutaneous electrical nerve stimulation (TENS) on the nerves also enhanced neural motor control and paretic limb functions in stroke subjects. ${ }^{100}$

\section{Neuromuscular electrical stimulation}

NMES utilizes short external electrical pulses to excite the peripheral nerves by modulating neuron hyperpolarization or depolarization. It generates muscle contractions through the skin surface, percutaneous or implanted electrodes. ${ }^{13}$ Typical NMES parameters include the pulse frequency (10 to $100 \mathrm{~Hz}$ ), ampli-

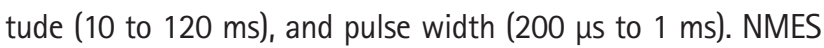
of higher frequencies generates larger forces, but quickly leads to muscle fatigue and fast reduction of contraction force. ${ }^{101}$ Wider pulse widths induces more pronounced cortical and muscular responses. ${ }^{102}$

Although stroke subjects cannot voluntarily move their affected limbs or generate muscle contractions similar to healthy subjects, their spinal motor neurons are intact and excitable. ${ }^{103}$ NMES intervention provides a supplementary or replacement tool for stroke patients to move paretic limbs. ${ }^{104}$ Pioneering study in 1961 demonstrated the feasibility of FES applications in hemiplegic gait performance. ${ }^{105}$ Moreover, implanted NMES hand neuroprosthesis was invented in 1988 for quadriplegic 
patients. ${ }^{106}$ NMES is effective in increasing muscle strength, relieving pain, decreasing muscle spasticity, and promoting poststroke motor control and physical rehabilitation. ${ }^{101,107,108} \mathrm{~A}$ closed-loop NMES system could also contribute to the motor recovery process. Here, cortical or muscular signals were used as control signals for either motor intention decoding or triggers. ${ }^{107,109-113}$ However, previous studies showed heterogeneous rehabilitation results with not enough subjects. Translation of the available research findings into clinical practice is still at its infancy. Several clinical trials have been conducted to examine the supplemental rehabilitation effects of NMES. For instance, systematic reviews and meta-analyses have concluded that EMG-NMES on upper limbs could promote functional recovery following chronic stroke and can readily be integrated into clinical practice. ${ }^{113}$ Future studies should conduct randomized control trials with larger sample size and with different patient characteristics to examine the rehabilitation efficiency of NMES in lower limb applications. More efficient stimulation protocols and rehabilitation strategies for individual subjects may further increase NMES therapeutic effects.

The underlying mechanisms of NMES in post-stroke motor recovery are only partially understood. Previously, motor stimulation has been focused to the muscle and motoneuron of the paretic limbs, and there is evidence that it could induce plasticity at the spinal levels. ${ }^{114} \mathrm{It}$ is proved recently that peripheral stimulation has central modulation effects. NMES also induces cortical plasticity by modulating the ascending pathways through the la muscle fiber afferents. ${ }^{102,115,116}$ Additionally, somatosensory inputs to the motor cortex are essential for motor learning and control, and play critical roles in the motor recovery process. ${ }^{100,117}$ NMES above the motor threshold increases excitability of corticomotor pathway by activating sensory axons and recruiting synaptic motoneurons and motor reflex. ${ }^{115}$ In a previous study, the cortico-muscular coherence in the NMES group was significantly higher in stroke patients when compared with the control group after 8 weeks NMES and motor training. ${ }^{100}$ Moreover, interaction of NMES in dynamic movements could facilitate understanding of post-stroke motor rehabilitation mechanisms in the physical world, and foster to its wide applications in stroke survivors. ${ }^{118}$

Previous studies primarily used standardized stimulation settings. It is necessary to investigate more optimized NMES paradigms considering muscle/cortical responses in different motor tasks and subjects. Recent progress in $\mathrm{BCl}$ could also assist NMES-based prosthetic systems through the brain. ${ }^{119}$ Additional$l y$, advancements of HD noninvasive NMES system in tetraplegia could allow for precise motor control of hand movement, and further benefit stroke patients. ${ }^{120}$ The latest inventions in electri- cal muscle stimulation including the self-powered triboelectric nanogenerator could facilitate deployment of sustainable therapeutic interventions. ${ }^{121}$ However, randomized controlled trials are needed to evaluate the clinical reliability of such therapeutic interventions. Through deliberate efforts, these techniques could be translated into practical clinical applications.

\section{Transcutaneous electrical nerve stimulation}

From the early 1970s, TENS has been extensively used for pain relief by modulating the descending pain inhibitory systems. ${ }^{122}$ In addition, TENS could effectively facilitate functional performance in hemiplegic patients ${ }^{123}$ and sensorimotor function restoration in chronic stroke patients. ${ }^{124}$ When TENS was combined with TOT in a randomized clinical trial, it enhanced voluntary lower limb movement for chronic stroke subjects. ${ }^{125}$ Moreover, home-based TENS with trunk training increased trunk muscle strength and motor control after stroke. ${ }^{126}$ Sensory stimulation with TENS promoted motor recovery therapeutic effects when combined with active rehabilitation training, the force production of ankle dorsiflexors was enhanced. ${ }^{127}$ Stimulation over peripheral nerves induced sensation along nerves and activated the related cortical area. Furthermore, a recent study showed that bilateral TENS applied over common peroneal nerve combined with TOT was superior to unilateral TENS with TOT in stroke paretic ankle dorsiflexion tasks. ${ }^{128}$ However, no consensus was reached owing to contradictory rehabilitation results across different TENS intensity. This necessitates evaluation of the underlying therapeutic mechanisms and optimization of efficient stimulus settings. ${ }^{129}$

A previous study manifested that cortical neuroplasticity could be induced by sensory input of TENS, which further influenced functional reorganization in brain regions adjacent to the stroke lesion. ${ }^{130}$ Decreased hyperexcitability of alpha motor neurons producing spastic ankle plantarflexor movement resulted from enhanced presynaptic inhibition after TENS. ${ }^{131}$ Similarly, reduction of intracortical inhibition was reported after TENS, with significant enhancement in upper limb functional score. ${ }^{132}$ Additionally, a 40-minute TENS over paretic median nerve can enhance gamma-band cortico-muscular coupling strength and modulate the CST. ${ }^{133} \mathrm{~A}$ recent fNIRS study showed that median nerve electrical stimulation induced ipsilesional prefrontal functional network changes and enhanced residual functions of paretic hands. ${ }^{134}$ Nevertheless, the detailed TENS modulatory mechanisms in motor recovery are still limited. This calls for further studies to elucidate the modulation mechanisms of neuroplasticity. In addition, randomized controlled trials with larger sample sizes should be conducted to assess the therapeutic effects of TENS. 


\section{Emerging electrical stimulation techniques}

In addition to NIBS and peripheral electrical stimulation protocols, invasive neurostimulation techniques emerged as potential rehabilitation strategies in the recent decade. ${ }^{135}$ The current invasive neurostimulation strategies for improving post-stroke motor recovery are mainly based on preliminary animal models. Thus, further research is necessary to test the clinical performance of such invasive neurostimulation strategies. Similar to noninvasive neurostimulation modalities, invasive stimulation tools also harness neuroplasticity, facilitate functional reorganization of brain regions, and ultimately promote clinical improvements of contralateral paretic limbs. Representative invasive neurostimulation modalities are summarized in the following section, including deep brain stimulation (DBS), epidural electrical stimulation, and vagus nerve stimulation (VNS).

\section{Deep brain stimulation}

DBS utilizes stimulating electrodes implanted deep into the brain. DBS was previously used to treat various movement disorders including essential tremor, Parkinson's disease, dystonia, and other related symptoms. ${ }^{136}$ It modulates local or remote brain regions depending on the parameter and target settings. DBS stimulates impaired neural circuits, thereby enhancing cortical network plasticity and facilitating functional reorganization of the perilesional cortex. ${ }^{137}$

As for post-stroke related motor deficits, DBS-based poststroke rehabilitation directly modulates deep brain regions which has shown great promise in resolving the limitations of previous noninvasive electrical stimulation settings. Though lacking systematic randomized clinical trials and conclusive explanation, for a stroke patient with motor weakness and spasticity, voluntary upper limb movement was improved following DBS intervention. ${ }^{138}$ Targeted stimulation was applied at posterior limb of the internal capsule (PLIC) or its neighboring area, where somatotopically organized CST fibers descend. PLIC transfers cortical information from M1 to motor neurons in the spinal cord. ${ }^{139}$ Therefore, DBS at PLIC possibly activated the descending neurons and further facilitate motor rehabilitation. Medial interhemispheric fissure area is responsible for lower limb cortical control, some of lower limb related cortical regions are deep inside and cannot be easily stimulated using noninvasive stimulation modalities. Invasive DBS could therefore be useful in recovering motor functions in stroke patients with lower limb impairment. ${ }^{140}$

The cerebral cortex and cerebellum are connected through the cerebro-ponto-cerebellar (CPC) and dentatothalamocorti- cal (DTC) pathways. Cross cerebellar diaschisis results from CPC tract disruption following a stroke, with an impact on residual motor functions. ${ }^{141}$ Dentate nucleus, the largest deep cerebellar nuclei, receives input from the lateral cerebellar hemisphere and CPC tract. Its primary outputs are transferred to the thalamus and the motor regions through the DTC tract. ${ }^{142}$ Cerebellar DBS at the dentate nucleus manipulates the DTC pathway, thereby facilitating motor recovery following ischemic stroke. ${ }^{143}$ Wathen et al. ${ }^{144}$ reviewed the latest advancements, theoretical foundations, rodent preclinical experiments, and current clinical trials in cerebellar DBS-based motor recovery following ischemic stroke. Results from preclinical and Phase I clinical trial underscored a therapeutic role of cerebellar DBS. Advanced Phase II and Phase III human clinical trials are needed to validate this effect. Moreover, the underlying modulation mechanisms of DBS should be illustrated exhaustively. A closed-loop DBS system could allow for real-time measurement of neurophysiological properties with enhanced precision of electrical stimulation.

Despite demonstrated benefit of DBS therapy, its application in post-stroke motor recovery is still limited owing to the risk involved in invasive surgery. Recent advancements in noninvasive DBS via temporally interfering electric fields stimulate deep neurons in the brain of a living mouse. ${ }^{145}$ Such noninvasive DBS could pave the way for potential treatment of poststroke motor recovery and other movement disorders. ${ }^{146}$ Future studies should explore new techniques and translate them into practical applications.

\section{Epidural cortical stimulation}

One of the main limitations of NIBS is that only about $25 \%$ of current penetrates deep into the brain to induce cortical excitability changes. The rest of the current is attenuated by the skin, skull, and subcutaneous tissues. ${ }^{44}$ This reduces the resolution and efficiency of stimulation. Invasive stimulation addresses this challenge by delivering currents directly to the ipsilesional periinfarct cortices like the M1, with modulatory effects similar to that of noninvasive tES modalities. Neuroplasticity could be enhanced through electrical neurostimulation, further inducing neuronal reorganization and functional improvements. Epidural cortical stimulation (ECS) has been paired concurrently with physical rehabilitation training to foster stroke functional recovery. ${ }^{135}$ ECS applied on ipsilesional brain in rodent models, Phase I and II clinical trials have demonstrated its safety and efficacy in motor recovery. ${ }^{147-149}$ However, Phase III clinical trials did not show significant functional score improvement. ${ }^{150}$ This should be attributed to the diverse stimulation site, lesion geometry, the inherent differences between animal and human experiments, 
and inviable descending motor pathways. ${ }^{151}$ Intact corticothalamic or CST fibers are essential factors influencing stimulation efficacy. Future studies should optimize electrical distribution and timing of stimulation.

There is no consensus on the placement of epidural stimulation. In rats with CST lesion, epidural stimulation on contralesional $\mathrm{M} 1$ restored motor functions by promoting CST sprouting based ipsilateral control..$^{152}$ Moreover, premotor stimulation could be an alternative target for impaired M1. ${ }^{153}$ In an ischemic rodent model, distributed stimulation showed better motor recovery compared to focal M1 stimulation. ${ }^{154}$ Such inconsistent stimulation placement settings might relate to the unclear rationale behind. Progress in microelectrode arrays underpins the prospective application in motor recovery with precise stimulation and real-time neurophysiological monitoring. ${ }^{155,156}$ This highlights crucial factors influencing post-stroke rehabilitation. Subsequent studies should employ the new techniques in post-stroke motor restoration.

\section{Vagus nerve stimulation}

Vagus nerve regulates different physiological functions and pathways, including inflammation, cerebral blood flow, glutamate excitotoxicity, and other neurotrophic processes. ${ }^{157}$ The vagus nerve comprises $80 \%$ sensory afferent fibers that carry information from the peripheral system to the brain, and 20\% motor efferent fibers that perform autonomous functions. ${ }^{158}$ Several complex cascades of processes in early stroke are influenced by afferent and efferent pathways of the vagus nerve. ${ }^{159}$ VNS is a potential tool for subacute stroke recovery owing to its anti-inflammatory and neuromodulators releasing properties. ${ }^{160,161}$

Invasive VNS is normally 0.25 to $3 \mathrm{~mA}$, equipped with bipolar electrodes placed underneath the chest skin and the left vagus nerve. ${ }^{162}$ Studies with ischemic rat models have demonstrated safety and feasibility of using VNS in post-stroke motor recovery. ${ }^{163,164}$ Coupled with rehabilitation training, VNS significantly promoted forelimb functional movement. ${ }^{164,165}$ VNS was also effective in facilitating long-lasting recovery and structural plasticity in corticospinal motor networks in rat models, with a resultant increased connectivity to forelimb muscles. ${ }^{166}$ Moreover, noninvasive VNS on cervical vagus nerve significantly decreased infarct volume, enhanced clinical scores and strength of forelimb grip following middle cerebral artery occlusion in rat models. ${ }^{167}$ The aforementioned VNS studies were still in preliminary stages and focused on animal models with small sample sizes. Further studies are required to investigate the functional role of VNS in motor restoration and validate its therapeutic effects in human.

\section{Summary and future directions}

Electrical stimulation has been widely applied to facilitate post-stroke motor recovery, but the modulatory mechanisms are not fully understood yet. Electrical stimulation manipulates corresponding neuronal circuits, which induces neuroplasticity changes that correlate with functional motor improvement. This nascent review lays the foundation for harnessing neuroplasticity of prospective electrical stimulation techniques to restore motor functions in stroke patients. NIBS and peripheral electrical stimulation are most frequently applied. Among the central-oriented approaches, NIBS protocols are the most convenient cortical stimulation, but their applications are limited by low stimulation resolution, non-optimized stimulation settings, and inter-subject variability. Peripheral electrical stimulation on the muscles and nerves induces corticospinal neuroplasticity, which influences cortical reorganization and functional recovery. Going forward, the performance of these two stimulation protocols requires further development. Specifically, optimized stimulation settings should be explored to enhance the motor recovery efficiency. Other emerging techniques, such as invasive brain stimulation tools for DBS and epidural stimulation, are limited by the high surgical risks to human stroke subjects, but its development would indeed address the limitation of noninvasive settings. Currently, protocols that accelerate post-stroke motor recovery by vagus stimulation are still in the elementary preclinical studies and worth efforts subsequently.

The recovery of motor function after stroke is influenced by the timing, targeting, stimulation intensity, other stimulation parameter settings, suitable experimental designs, and task-specificity. Rational combination of different stimulation protocols may yield better clinical outcomes, such as PAS integrating cortical and peripheral stimulation. Further, precision medicine incorporating patient-tailored stimulation and rehabilitation training might be more effective in motor rehabilitation. The development of precise and flexible computational models of electrical stimulation modalities can facilitate understanding of current flow and refining electrotherapy designs. The latest technological advancements, such as self-powered, high density microelectrodes, and the minimally invasive electrical stimulation tools, indicate more precise and localized stimulation modalities. Additionally, measurements with adequate spatial and temporal resolution may reveal the neurophysiological properties during/following electrical stimulation and the underlying motor control and recovery mechanisms. Thus, closed-loop electrical stimulation with neural feedback provides higher temporal resolution and real-time control, whereas optimal artifact removal algo- 
rithms might be pivotal for such systems. Lastly, all the stimulation devices and protocols should be guaranteed safe and well-tolerated in practical applications.

\section{Conclusions}

Electrical stimulation protocols have shown great clinical potential in post-stroke motor recovery. More precise and effective motor restoration strategies may further benefit individual stroke subjects. Subsequent studies should expand the detailed modulatory mechanisms of the existing modalities and translate the state-of-art techniques to improve the treatment of stroke survivors and people with other movement disorders.

\section{Disclosure}

The authors declare no potential conflict of interest.

\section{Acknowledgments}

This study is supported by Hong Kong Research Grant Council (GRF No: 14208118), Hong Kong.

\section{References}

1. Pandian JD, Gall SL, Kate MP, Silva GS, Akinyemi RO, Ovbiagele $\mathrm{BI}$, et al. Prevention of stroke: a global perspective. Lancet 2018;392:1269-1278.

2. Feigin VL, Krishnamurthi RV, Parmar P, Norrving B, Mensah $\mathrm{GA}$, Bennett DA, et al. Update on the global burden of ischemic and hemorrhagic stroke in 1990-2013: the GBD 2013 study. Neuroepidemiology 2015;45:161-176.

3. Hatem SM, Saussez G, Della Faille M, Prist V, Zhang X, Dispa $D$, et al. Rehabilitation of motor function after stroke: a multiple systematic review focused on techniques to stimulate upper extremity recovery. Front Hum Neurosci 2016;10:442.

4. Lodha N, Harrell J, Eisenschenk S, Christou EA. Motor impairments in transient ischemic attack increase the odds of a subsequent stroke: a meta-analysis. Front Neurol 2017;8:243.

5. Singam A, Ytterberg C, Tham $K_{1}$ von Koch L. Participation in complex and social everyday activities six years after stroke: predictors for return to pre-stroke level. PLoS One 2015;10: e0144344.

6. Shumway-Cook A, Woollacott MH. Motor Control: Translating Research into Clinical Practice. 5th ed. Philadelphia, PA: Wolters Kluwer Health, 2016.

7. Scott SH. Optimal feedback control and the neural basis of volitional motor control. Nat Rev Neurosci 2004;5:532-546.
8. Kandel ER, Schwartz JH, Jessell TM; Department of Biochemistry and Molecular Biophysics, Siegelbaum SA, Hudspeth AJ. Principles of Neural Science. 5th ed. New York, NY: McGrawHill Publishing, 2012.

9. Baker SN, Zaaimi B, Fisher KM, Edgley SA, Soteropoulos DS. Pathways mediating functional recovery. Prog Brain Res 2015;218:389-412.

10. Rathelot JA, Strick PL. Subdivisions of primary motor cortex based on cortico-motoneuronal cells. Proc Natl Acad Sci U S A 2009;106:918-923.

11. Sakai ST, Davidson AG, Buford JA. Reticulospinal neurons in the pontomedullary reticular formation of the monkey (Macaca fascicularis). Neuroscience 2009;163:1158-1170.

12. Krakauer JW. Motor learning: its relevance to stroke recovery and neurorehabilitation. Curr Opin Neurol 2006;19:84-90.

13. Takeda K, Tanino G, Miyasaka H. Review of devices used in neuromuscular electrical stimulation for stroke rehabilitation. Med Devices (Auckl) 2017;10:207-213.

14. Hebb D0. The Organization of Behavior a Neuropsychological Theory. New York, NY: John Wiley and Sons, 1949.

15. Krakauer JW, Carmichael ST. Broken Movement: The Neurobiology of Motor Recovery after Stroke. Cambridge, MA: The MIT Press, 2017.

16. Li S. Spasticity, motor recovery, and neural plasticity after stroke. Front Neurol 2017;8:120.

17. French B, Thomas LH, Coupe J, McMahon NE, Connell L, Harrison J, et al. Repetitive task training for improving functional ability after stroke. Cochrane Database Syst Rev 2016;11: CD006073.

18. Minassian K, Hofstoetter U, Tansey K, Mayr W. Neuromodulation of lower limb motor control in restorative neurology. Clin Neurol Neurosurg 2012;114:489-497.

19. Young W. Electrical stimulation and motor recovery. Cell Transplant 2015;24:429-446.

20. O'Brien AT, Bertolucci F, Torrealba-Acosta G, Huerta R, Fregni F, Thibaut A. Non-invasive brain stimulation for fine motor improvement after stroke: a meta-analysis. Eur J Neurol 2018;25: 1017-1026.

21. Polanía $R$, Nitsche MA, Ruff CC. Studying and modifying brain function with non-invasive brain stimulation. Nat Neurosci 2018;21:174-187.

22. Kang N, Summers JJ, Cauraugh JH. Transcranial direct current stimulation facilitates motor learning post-stroke: a systematic review and meta-analysis. J Neurol Neurosurg Psychiatry 2016;87:345-355.

23. Allman C, Amadi U, Winkler AM, Wilkins L, Filippini N, Kischka $U$, et al. Ipsilesional anodal tDCS enhances the functional benefits of rehabilitation in patients after stroke. Sci Trans/ 
Med 2016;8:330re1.

24. Brunoni A, Nitsche M, Loo C. Transcranial Direct Current Stimulation in Neuropsychiatric Disorders. Cham, CH: Springer International Publishing, 2016.

25. Bindman $\sqcup$, Lippold OC, Redfearn JW. Long-lasting changes in the level of the electrical activity of the cerebral cortex produced bypolarizing currents. Nature 1962;196:584-585.

26. Priori A, Berardelli A, Rona S, Accornero N, Manfredi M. Polarization of the human motor cortex through the scalp. Neuroreport 1998;9:2257-2260.

27. Nitsche MA, Paulus W. Excitability changes induced in the human motor cortex by weak transcranial direct current stimulation. J Physiol 2000;527 Pt 3:633-639.

28. Borckardt JJ, Bikson M, Frohman H, Reeves ST, Datta A, Bansal $V$, et al. A pilot study of the tolerability and effects of high-definition transcranial direct current stimulation (HDtDCS) on pain perception. J Pain 2012;13:112-120.

29. Woods AJ, Antal A, Bikson M, Boggio PS, Brunoni AR, Celnik $P$, et al. A technical guide to tDCS, and related non-invasive brain stimulation tools. Clin Neurophysiol 2016;127:10311048.

30. Antal A, Boros K, Poreisz C, Chaieb L, Terney D, Paulus W. Comparatively weak after-effects of transcranial alternating current stimulation (tACS) on cortical excitability in humans. Brain Stimul 2008;1:97-105.

31. Krause V, Wach C, Südmeyer M, Ferrea S, Schnitzler A, Pollok B. Cortico-muscular coupling and motor performance are modulated by $20 \mathrm{~Hz}$ transcranial alternating current stimulation (tACS) in Parkinson's disease. Front Hum Neurosci 2014;7: 928.

32. Chaieb L, Antal A, Paulus W. Transcranial alternating current stimulation in the low $\mathrm{kHz}$ range increases motor cortex excitability. Restor Neurol Neurosci 2011;29:167-175.

33. Suzuki K, Fujiwara T, Tanaka N, Tsuji T, Masakado Y, Hase K, et al. Comparison of the after-effects of transcranial direct current stimulation over the motor cortex in patients with stroke and healthy volunteers. Int J Neurosci 2012;122:675681.

34. Li LM, Violante IR, Leech R, Ross E, Hampshire A, Opitz A, et al. Brain state and polarity dependent modulation of brain networks by transcranial direct current stimulation. Hum Brain Mapp 2019;40:904-915.

35. Cao J, Wang $X$, Liu $H$, Alexandrakis G. Directional changes in information flow between human brain cortical regions after application of anodal transcranial direct current stimulation (tDCS) over Broca's area. Biomed Opt Express 2018;9:52965317.

36. Soekadar SR, Witkowski M, Cossio EG, Birbaumer N, Robin- son $\mathrm{SE}$, Cohen LG. In vivo assessment of human brain oscillations during application of transcranial electric currents. Nat Commun 2013;4:2032.

37. Baxter BS, Edelman BJ, Nesbitt N, He B. Sensorimotor rhythm $\mathrm{BCl}$ with simultaneous high definition-transcranial direct current stimulation alters task performance. Brain Stimul 2016;9:834-841.

38. Krause MR, Zanos TP, Csorba BA, Pilly PK, Choe J, Phillips ME, et al. Transcranial direct current stimulation facilitates associative learning and alters functional connectivity in the primate brain. Curr Biol 2017;27:3086-3096.

39. Tanaka S, Hanakawa T, Honda M, Watanabe K. Enhancement of pinch force in the lower leg by anodal transcranial direct current stimulation. Exp Brain Res 2009;196:459-465.

40. Madhavan S, Weber KA 2nd, Stinear JW. Non-invasive brain stimulation enhances fine motor control of the hemiparetic ankle: implications for rehabilitation. Exp Brain Res 2011;209: 9-17.

41. Pan L, Zhang D, Sheng $X$, Zhu X. Improving myoelectric control for amputees through transcranial direct current stimulation. IEEE Trans Biomed Eng 2015;62:1927-1936.

42. Polanía R, Nitsche MA, Paulus W. Modulating functional connectivity patterns and topological functional organization of the human brain with transcranial direct current stimulation. Hum Brain Mapp 2011;32:1236-1249.

43. Vecchio F, Pellicciari MC, Miraglia F, Brignani D, Miniussi C, Rossini PM. Effects of transcranial direct current stimulation on the functional coupling of the sensorimotor cortical network. Neuroimage 2016;140:50-56.

44. Vöröslakos M, Takeuchi Y, Brinyiczki K, Zombori T, Oliva A, Fernández-Ruiz $A$, et al. Direct effects of transcranial electric stimulation on brain circuits in rats and humans. Nat Commun 2018;9:483.

45. Bao SC, Wong WW, Leung TWH, Tong KY. Cortico-muscular coherence modulated by high-definition transcranial direct current stimulation in people with chronic stroke. IEEE Trans Neural Syst Rehabil Eng 2019;27:304-313.

46. Hong X, Lu ZK, Teh I, Nasrallah FA, Teo WP, Ang KK, et al. Brain plasticity following $\mathrm{Ml}-\mathrm{BCl}$ training combined with tDCS in a randomized trial in chronic subcortical stroke subjects: a preliminary study. Sci Rep 2017;7:9222.

47. Gellner AK, Reis J, Holtick C, Schubert C, Fritsch B. Direct current stimulation-induced synaptic plasticity in the sensorimotor cortex: structure follows function. Brain Stimul 2020;13: 80-88.

48. Otal B, Dutta A, Foerster Á, Ripolles O, Kuceyeski A, Miranda $P C$, et al. Opportunities for guided multichannel non-invasive transcranial current stimulation in poststroke rehabilitation. 
Front Neurol 2016;7:21.

49. Hummel F, Cohen LG. Improvement of motor function with noninvasive cortical stimulation in a patient with chronic stroke. Neurorehabil Neural Repair 2005;19:14-19.

50. Schlaug G, Renga V, Nair D. Transcranial direct current stimulation in stroke recovery. Arch Neurol 2008;65:1571-1576.

51. Gomez Palacio Schjetnan A, Faraji J, Metz GA, Tatsuno M, Luczak A. Transcranial direct current stimulation in stroke rehabilitation: a review of recent advancements. Stroke Res Treat 2013;2013:170256.

52. Hummel FC, Cohen LG. Non-invasive brain stimulation: a new strategy to improve neurorehabilitation after stroke? Lancet Neurol 2006;5:708-712.

53. Di Pino G, Pellegrino G, Assenza G, Capone F, Ferreri F, Formica $D$, et al. Modulation of brain plasticity in stroke: a novel model for neurorehabilitation. Nat Rev Neurol 2014;10:597608.

54. Chieffo R, Comi G, Leocani L. Noninvasive neuromodulation in poststroke gait disorders: rationale, feasibility, and state of the art. Neurorehabil Neural Repair 2016;30:71-82.

55. Lefaucheur JP, Antal A, Ayache SS, Benninger DH, Brunelin J, Cogiamanian $F$, et al. Evidence-based guidelines on the therapeutic use of transcranial direct current stimulation (tDCS). Clin Neurophysiol 2017;128:56-92.

56. Malerba P, Straudi S, Fregni F, Bazhenov M, Basaglia N. Using biophysical models to understand the effect of tDCS on neurorehabilitation: searching for optimal covariates to enhance poststroke recovery. Front Neurol 2017;8:58.

57. Fischer DB, Fried PJ, Ruffini G, Ripolles O, Salvador R, Banus $J$, et al. Multifocal tDCS targeting the resting state motor network increases cortical excitability beyond traditional tDCS targeting unilateral motor cortex. Neuroimage 2017; 157:34-44.

58. Truong DQ, Magerowski G, Blackburn GL, Bikson M, AlonsoAlonso M. Computational modeling of transcranial direct current stimulation (tDCS) in obesity: impact of head fat and dose guidelines. Neuroimage Clin 2013;2:759-766.

59. Leite J, Morales-Quezada L, Carvalho S, Thibaut A, Doruk D, Chen $C F$, et al. Surface eeg-transcranial direct current stimulation (tDCS) closed-loop system. Int J Neural Syst 2017;27: 1750026.

60. Zhou A, Johnson BC, Muller R. Toward true closed-loop neuromodulation: artifact-free recording during stimulation. Curr Opin Neurobiol 2018;50:119-127.

61. Koganemaru S, Kitatani R, Fukushima-Maeda A, Mikami Y, Okita $Y$, Matsuhashi $M$, et al. Gait-synchronized rhythmic brain stimulation improves poststroke gait disturbance: a pilot study. Stroke 2019;50:3205-3212.
62. Rotenberg A, Horvath JC, Pascual-Leone A. Transcranial Magnetic Stimulation. New York, NY: Humana Press, 2014.

63. Barker AT, Jalinous R, Freeston IL. Non-invasive magnetic stimulation of human motor cortex. Lancet 1985;1:1106-1107.

64. Hoyer EH, Celnik PA. Understanding and enhancing motor recovery after stroke using transcranial magnetic stimulation. Restor Neurol Neurosci 2011;29:395-409.

65. Trompetto C, Assini A, Buccolieri A, Marchese R, Abbruzzese G. Motor recovery following stroke: a transcranial magnetic stimulation study. Clin Neurophysiol 2000;111:1860-1867.

66. Reis J, Swayne OB, Vandermeeren Y, Camus M, Dimyan MA, Harris-Love $M$, et al. Contribution of transcranial magnetic stimulation to the understanding of cortical mechanisms involved in motor control. J Physiol 2008;586:325-351.

67. Maeda F, Keenan JP, Tormos JM, Topka H, Pascual-Leone A. Interindividual variability of the modulatory effects of repetitive transcranial magnetic stimulation on cortical excitability. Exp Brain Res 2000;133:425-430.

68. Takeuchi N, Chuma T, Matsuo Y, Watanabe I, Ikoma K. Repetitive transcranial magnetic stimulation of contralesional primary motor cortex improves hand function after stroke. Stroke 2005;36:2681-2686.

69. Sung WH, Wang CP, Chou CL, Chen YC, Chang YC, Tsai PY. Efficacy of coupling inhibitory and facilitatory repetitive transcranial magnetic stimulation to enhance motor recovery in hemiplegic stroke patients. Stroke 2013;44:1375-1382.

70. Dimyan MA, Cohen LG. Neuroplasticity in the context of motor rehabilitation after stroke. Nat Rev Neurol 2011;7:76-85.

71. Dobek CE, Blumberger DM, Downar J, Daskalakis Z, Vila-Rodriguez $\mathrm{F}$. Risk of seizures in transcranial magnetic stimulation: a clinical review to inform consent process focused on bupropion. Neuropsychiatr Dis Treat 2015;11:2975-2987.

72. Huang YZ, Chen RS, Rothwell JC, Wen HY. The after-effect of human theta burst stimulation is NMDA receptor dependent. Clin Neurophysiol 2007;118:1028-1032.

73. Carson RG, Kennedy NC. Modulation of human corticospinal excitability by paired associative stimulation. Front Hum Neurosci 2013;7:823.

74. Fratello F, Veniero D, Curcio G, Ferrara M, Marzano C, Moroni $F_{1}$ et al. Modulation of corticospinal excitability by paired associative stimulation: reproducibility of effects and intraindividual reliability. Clin Neurophysiol 2006;117:2667-2674.

75. Bates KA, Rodger J. Repetitive transcranial magnetic stimulation for stroke rehabilitation-potential therapy or misplaced hope? Restor Neurol Neurosci 2015;33:557-569.

76. Hao Z, Wang D, Zeng Y, Liu M. Repetitive transcranial magnetic stimulation for improving function after stroke. Cochrane Database Syst Rev 2013;5:CD008862. 
77. Malcolm MP, Triggs WJ, Light KE, Gonzalez Rothi $\sqcup$, Wu S, Reid $K$, et al. Repetitive transcranial magnetic stimulation as an adjunct to constraint-induced therapy: an exploratory randomized controlled trial. Am J Phys Med Rehabil 2007;86:707715.

78. Cárdenas-Morales L, Volz $\sqcup$, Michely J, Rehme AK, Pool EM,

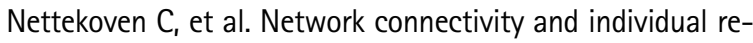
sponses to brain stimulation in the human motor system. Cereb Cortex 2014;24:1697-1707.

79. Tremblay S, Rogasch NC, Premoli I, Blumberger DM, Casarotto $S$, Chen $R$, et al. Clinical utility and prospective of TMSEEG. Clin Neurophysiol 2019;130:802-844.

80. Kawasaki M, Uno Y, Mori J, Kobata K, Kitajo K. Transcranial magnetic stimulation-induced global propagation of transient phase resetting associated with directional information flow. Front Hum Neurosci 2014;8:173.

81. Valero-Cabré $A$, Amengual $J$, Stengel $C$, Pascual-Leone $A$, Coubard OA. Transcranial magnetic stimulation in basic and clinical neuroscience: a comprehensive review of fundamental principles and novel insights. Neurosci Biobehav Rev 2017;83:381-404.

82. Koponen LM, Nieminen JO, Ilmoniemi RJ. Multi-locus transcranial magnetic stimulation-theory and implementation. Brain Stimul 2018;11:849-855.

83. Manto $M$, Bower JM, Conforto AB, Delgado-García JM, da Guarda SN, Gerwig M, et al. Consensus paper: roles of the cerebellum in motor control: the diversity of ideas on cerebellar involvement in movement. Cerebellum 2012;11:457-487.

84. Bostan AC, Dum RP, Strick PL. Cerebellar networks with the cerebral cortex and basal ganglia. Trends Cogn Sci 2013;17: 241-254.

85. Hamada M, Strigaro G, Murase N, Sadnicka A, Galea JM, Edwards $\mathrm{MJ}$, et al. Cerebellar modulation of human associative plasticity. J Physiol 2012;590:2365-2374.

86. Carey MR. Synaptic mechanisms of sensorimotor learning in the cerebellum. Curr Opin Neurobiol 2011;21:609-615.

87. Wessel MJ, Hummel FC. Non-invasive cerebellar stimulation: a promising approach for stroke recovery? Cerebellum 2018; 17:359-371.

88. Galea JM, Jayaram G, Ajagbe L, Celnik P. Modulation of cerebellar excitability by polarity-specific noninvasive direct current stimulation. J Neurosci 2009;29:9115-9122.

89. Erfmann KLC. The influence of cerebellar transcranial direct current stimulation (tDCS) on motor skill learning in swallowing. https://pdfs.semanticscholar.org/279b/a59625b6b40e8e094969c14ce476405eb849.pdf. 2018. Accessed January 19, 2020.

90. Doppelmayr M, Pixa NH, Steinberg F. Cerebellar, but not mo- tor or parietal, high-density anodal transcranial direct current stimulation facilitates motor adaptation. J Int Neuropsychol Soc 2016;22:928-936.

91. Zandvliet SB, Meskers CGM, Kwakkel G, van Wegen EEH. Short-term effects of cerebellar tDCS on standing balance performance in patients with chronic stroke and healthy age-matched elderly. Cerebellum 2018;17:575-589.

92. Zandvliet SB, Meskers CG, Nijland RH, Daffertshofer A, Kwakkel $G$, van Wegen EE. The effect of cerebellar transcranial direct current stimulation to improve standing balance performance early post-stroke, study protocol of a randomized controlled trial. Int J Stroke 2019;14:650-657.

93. Naro A, Leo A, Russo M, Cannavò A, Milardi D, Bramanti $P$, et al. Does transcranial alternating current stimulation induce cerebellum plasticity? Feasibility, safety and efficacy of a novel electrophysiological approach. Brain Stimul 2016;9:388-395.

94. Iglesias C, Nielsen JB, Marchand-Pauvert V. Corticospinal inhibition of transmission in propriospinal-like neurones during human walking. Eur J Neurosci 2008;28:1351-1361.

95. Cogiamanian F, Vergari M, Pulecchi F, Marceglia S, Priori A. Effect of spinal transcutaneous direct current stimulation on somatosensory evoked potentials in humans. Clin Neurophysiol 2008;119:2636-2640.

96. Ahmed Z. Trans-spinal direct current stimulation modulates motor cortex-induced muscle contraction in mice. J Appl Physiol (1985) 2011;110:1414-1424.

97. Picelli A, Chemello $E$, Castellazzi $P$, Roncari $L$, Waldner $A$,

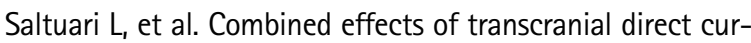
rent stimulation (tDCS) and transcutaneous spinal direct current stimulation (tsDCS) on robot-assisted gait training in patients with chronic stroke: a pilot, double blind, randomized controlled trial. Restor Neurol Neurosci 2015;33:357368.

98. Picelli $A$, Chemello $E$, Castellazzi P, Filippetti $M$, Brugnera $A$, Gandolfi $M$, et al. Combined effects of cerebellar transcranial direct current stimulation and transcutaneous spinal direct current stimulation on robot-assisted gait training in patients with chronic brain stroke: a pilot, single blind, randomized controlled trial. Restor Neurol Neurosci 2018;36: 161-171.

99. Picelli A, Brugnera A, Filippetti M, Mattiuz N, Chemello $E_{\text {, }}$ Modenese $A$, et al. Effects of two different protocols of cerebellar transcranial direct current stimulation combined with transcutaneous spinal direct current stimulation on robotassisted gait training in patients with chronic supratentorial stroke: a single blind, randomized controlled trial. Restor Neurol Neurosci 2019;37:97-107.

100. Pan LH, Yang WW, Kao CL, Tsai MW, Wei SH, Fregni $F$, et al. 
Effects of 8-week sensory electrical stimulation combined with motor training on EEG-EMG coherence and motor function in individuals with stroke. Sci Rep 2018;8:9217.

101. Sheffler $L R$, Chae J. Neuromuscular electrical stimulation in neurorehabilitation. Muscle Nerve 2007;35:562-590.

102. Collins DF. Central contributions to contractions evoked by tetanic neuromuscular electrical stimulation. Exerc Sport Sci Rev 2007;35:102-109.

103. Lynch $C_{L}$, Popovic MR. Functional electrical stimulation. IEEE Control Syst Mag 2008;28:40-50.

104. Veldman MP, Maurits NM, Zijdewind I, Maffiuletti NA, van Middelkoop S, Mizelle JC, et al. Somatosensory electrical stimulation improves skill acquisition, consolidation, and transfer by increasing sensorimotor activity and connectivity. J Neurophysiol 2018;120:281-290.

105. Liberson WT, Holmquest HJ, Scot D, Dow M. Functional electrotherapy: stimulation of the peroneal nerve synchronized with the swing phase of the gait of hemiplegic patients. Arch Phys Med Rehabil 1961;42:101-105.

106. Peckham PH, Keith MW, Freehafer AA. Restoration of functional control by electrical stimulation in the upper extremity of the quadriplegic patient. J Bone Joint Surg Am 1988;70: 144-148.

107. Hu XL, Tong RK, Ho NS, Xue JJ, Rong W, Li LS. Wrist rehabilitation assisted by an electromyography-driven neuromuscular electrical stimulation robot after stroke. Neurorehabil Neural Repair 2015;29:767-776.

108. Howlett OA, Lannin NA, Ada L, McKinstry C. Functional electrical stimulation improves activity after stroke: a systematic review with meta-analysis. Arch Phys Med Rehabil 2015;96: 934-943.

109. Meng $F_{1}$ Tong $K_{1}$ Chan S, Wong W, Lui $K_{1}$ Tang $K_{1}$ et al. BClFES training system design and implementation for rehabilitation of stroke patients. In: 2008 IEEE International Joint Conference on Neural Networks (IEEE World Congress on Computational Intelligence); 2008 Jun 1-8; Hong Kong, CN. Piscataway, NJ: IEEE; 2008.

110. Cauraugh J, Light K, Kim S, Thigpen M, Behrman A. Chronic motor dysfunction after stroke: recovering wrist and finger extension by electromyography-triggered neuromuscular stimulation. Stroke 2000;31:1360-1364.

111. Do AH, Wang PT, King CE, Abiri A, Nenadic Z. Brain-computer interface controlled functional electrical stimulation system for ankle movement. J Neuroeng Rehabil 2011;8:49.

112. Chung $E_{1}$ Kim JH, Park DS, Lee BH. Effects of brain-computer interface-based functional electrical stimulation on brain activation in stroke patients: a pilot randomized controlled trial. J Phys Ther Sci 2015;27:559-562.
113. Monte-Silva K, Piscitelli D, Norouzi-Gheidari N, Batalla MAP, Archambault P, Levin MF. Electromyogram-related neuromuscular electrical stimulation for restoring wrist and hand movement in poststroke hemiplegia: a systematic review and meta-analysis. Neurorehabil Neural Repair 2019;33:96-111.

114. Field-Fote EC. Electrical stimulation modifies spinal and cortical neural circuitry. Exerc Sport Sci Rev 2004;32:155-160.

115. Chipchase LS, Schabrun SM, Hodges PW. Peripheral electrical stimulation to induce cortical plasticity: a systematic review of stimulus parameters. Clin Neurophysiol 2011;122:456-463.

116. Wegrzyk J, Ranjeva JP, Fouré A, Kavounoudias A, Vilmen C, Mattei JP, et al. Specific brain activation patterns associated with two neuromuscular electrical stimulation protocols. Sci Rep 2017;7:2742.

117. Vidoni ED, Acerra NE, Dao E, Meehan SK, Boyd LA. Role of the primary somatosensory cortex in motor learning: an rTMS study. Neurobiol Learn Mem 2010;93:532-539.

118. Bao SC, Leung WC, K Cheung VC, Zhou P, Tong KY. Pathwayspecific modulatory effects of neuromuscular electrical stimulation during pedaling in chronic stroke survivors. J Neuroeng Rehabil 2019;16:143.

119. Bouton CE, Shaikhouni A, Annetta NV, Bockbrader MA, Friedenberg DA, Nielson DM, et al. Restoring cortical control of functional movement in a human with quadriplegia. Nature 2016;533:247-250.

120. Annetta NV, Friend J, Schimmoeller A, Buck VS, Friedenberg $D A$, Bouton $C E$, et al. A high definition noninvasive neuromuscular electrical stimulation system for cortical control of combinatorial rotary hand movements in a human with tetraplegia. IEEE Trans Biomed Eng 2019;66:910-919.

121. Wang J, Wang $H_{1}$ He $T$, He $B$, Thakor NV, Lee $C$. Investigation of low-current direct stimulation for rehabilitation treatment related to muscle function loss using self-powered TENG system. Adv Sci (Weinh) 2019;6:1900149.

122. Augustinsson $L E$, Bohlin $P$, Bundsen $P$, Carlsson $C A$, Forssman $L$, Sjöberg $P$, et al. Pain relief during delivery by transcutaneous electrical nerve stimulation. Pain 1977;4:59-65.

123. Tekeoğlu Y, Adak B, Göksoy T. Effect of transcutaneous electrical nerve stimulation (TENS) on Barthel Activities of Daily Living (ADL) index score following stroke. Clin Rehabil 1998;12: 277-280.

124. Peurala SH, Pitkänen K, Sivenius J, Tarkka IM. Cutaneous electrical stimulation may enhance sensorimotor recovery in chronic stroke. Clin Rehabil 2002;16:709-716.

125. Ng SS, Hui-Chan CW. Transcutaneous electrical nerve stimulation combined with task-related training improves lower limb functions in subjects with chronic stroke. Stroke 2007;38: 2953-2959. 
126. Chan BK, Ng SS, Ng GY. A home-based program of transcutaneous electrical nerve stimulation and task-related trunk training improves trunk control in patients with stroke: a randomized controlled clinical trial. Neurorehabil Neural Repair 2015;29:70-79.

127. Laufer $Y$, Elboim-Gabyzon M. Does sensory transcutaneous electrical stimulation enhance motor recovery following a stroke? A systematic review. Neurorehabil Neural Repair $2011 ; 25: 799-809$.

128. Kwong PWH, Ng GYF, Chung RCK, Ng SSM. Bilateral transcutaneous electrical nerve stimulation improves lower-limb motor function in subjects with chronic stroke: a randomized controlled trial. J Am Heart Assoc 2018;7:e007341.

129. Grant VM, Gibson A, Shields N. Somatosensory stimulation to improve hand and upper limb function after stroke-a systematic review with meta-analyses. Top Stroke Rehabil 2018;25: 150-160.

130. Liepert J, Bauder H, Wolfgang HR, Miltner WH, Taub E, WeiIler $\mathrm{C}$. Treatment-induced cortical reorganization after stroke in humans. Stroke 2000;31:1210-1216.

131. Levin MF, Hui-Chan CW. Relief of hemiparetic spasticity by TENS is associated with improvement in reflex and voluntary motor functions. Electroencephalogr Clin Neurophysiol 1992;85:131-142.

132. Celnik P, Hummel F, Harris-Love M, Wolk R, Cohen LG. Somatosensory stimulation enhances the effects of training functional hand tasks in patients with chronic stroke. Arch Phys Med Rehabil 2007;88:1369-1376.

133. Lai MI, Pan LL, Tsai MW, Shih YF, Wei SH, Chou LW. Investigating the effects of peripheral electrical stimulation on corticomuscular functional connectivity stroke survivors. Top Stroke Rehabil 2016;23:154-162.

134. Huo C, Li $X$, Jing J, Ma Y, Li W, Wang $Y$, et al. Median nerve electrical stimulation-induced changes in effective connectivity in patients with stroke as assessed with functional near-infrared spectroscopy. Neurorehabil Neural Repair 2019;33: 1008-1017.

135. Plow EB, Machado A. Invasive neurostimulation in stroke rehabilitation. Neurotherapeutics 2014;11:572-582.

136. Pycroft L, Stein J, Aziz T. Deep brain stimulation: an overview of history, methods, and future developments. Brain Neurosci Adv 2018;2:2398212818816017.

137. Lozano AM, Lipsman N. Probing and regulating dysfunctional circuits using deep brain stimulation. Neuron 2013;77:406424.

138. Phillips NI, Bhakta BB. Affect of deep brain stimulation on limb paresis after stroke. Lancet 2000;356:222-223.

139. Holodny Al, Gor DM, Watts R, Gutin PH, Ulug AM. Diffusion- tensor MR tractography of somatotopic organization of corticospinal tracts in the internal capsule: initial anatomic results in contradistinction to prior reports. Radiology 2005;234:649653.

140. Hunsche $S$, Sauner D, Runge MJ, Lenartz D, El Majdoub F,

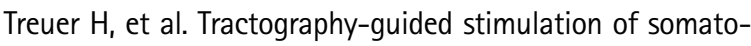
sensory fibers for thalamic pain relief. Stereotact Funct Neurosurg 2013;91:328-334.

141. Sommer WH, Bollwein $C$, Thierfelder KM, Baumann A, Janssen $H$, Ertl-Wagner $B$, et al. Crossed cerebellar diaschisis in patients with acute middle cerebral artery infarction: occurrence and perfusion characteristics. J Cereb Blood Flow Metab 2016;36:743-754.

142. Dum RP, Li C, Strick PL. Motor and nonmotor domains in the monkey dentate. Ann N Y Acad Sci 2002;978:289-301.

143. Machado A, Baker KB. Upside down crossed cerebellar diaschisis: proposing chronic stimulation of the dentatothalamocortical pathway for post-stroke motor recovery. Front Integr Neurosci 2012;6:20.

144. Wathen CA, Frizon LA, Maiti TK, Baker KB, Machado AG. Deep brain stimulation of the cerebellum for poststroke motor rehabilitation: from laboratory to clinical trial. Neurosurg Focus 2018;45:E13.

145. Grossman N, Bono D, Dedic N, Kodandaramaiah SB, Rudenko $A$, Suk HJ, et al. Noninvasive deep brain stimulation via temporally interfering electric fields. Cell 2017;169:1029-1041.

146. Grossman N, Okun MS, Boyden ES. Translating temporal interference brain stimulation to treat neurological and psychiatric conditions. JAMA Neurol 2018;75:1307-1308.

147. Brown JA, Lutsep HL, Weinand M, Cramer SC. Motor cortex stimulation for the enhancement of recovery from stroke: a prospective, multicenter safety study. Neurosurgery 2006; 58:464-473.

148. Adkins DL, Hsu JE, Jones TA. Motor cortical stimulation promotes synaptic plasticity and behavioral improvements following sensorimotor cortex lesions. Exp Neuro/ 2008;212:1428.

149. Levy R, Ruland S, Weinand M, Lowry D, Dafer R, Bakay R. Cortical stimulation for the rehabilitation of patients with hemiparetic stroke: a multicenter feasibility study of safety and efficacy. J Neurosurg 2008;108:707-714.

150. Levy RM, Harvey RL, Kissela BM, Winstein CJ, Lutsep HL, Parrish $\mathrm{TB}$, et al. Epidural electrical stimulation for stroke rehabilitation: results of the prospective, multicenter, randomized, single-blinded everest trial. Neurorehabil Neural Repair 2016;30:107-119.

151. Plow EB, Carey JR, Nudo RJ, Pascual-Leone A. Invasive cortical stimulation to promote recovery of function after stroke: 
a critical appraisal. Stroke 2009;40:1926-1931.

152. Carmel JB, Kimura $\mathrm{H}$, Martin JH. Electrical stimulation of motor cortex in the uninjured hemisphere after chronic unilateral injury promotes recovery of skilled locomotion through ipsilateral control. J Neurosci 2014;34:462-466.

153. Gao H, Yu C, Zhang J, Zhang S, Xu K. Effect of epidural electrical stimulation on severely affected forelimb reaching and grasping function. In: 2019 9th International IEEE/EMBS Conference on Neural Engineering (NER); 2019 Mar 20-23; San Francisco, CA. Piscataway, NJ: IEEE; 2019.

154. Boychuk JA, Adkins DL, Kleim JA. Distributed versus focal cortical stimulation to enhance motor function and motor map plasticity in a rodent model of ischemia. Neurorehabil Neural Repair 2011;25:88-97.

155. Yang J, Liu L, Li T, Li C. Array focal cortical stimulation enhances motor function recovery and brain remodeling in a rat model of ischemia. J Stroke Cerebrovasc Dis 2017;26:658665.

156. Patel SR, Lieber CM. Precision electronic medicine in the brain. Nat Biotechnol 2019;37:1007-1012.

157. Cheyuo C, Jacob A, Wu R, Zhou M, Coppa GF, Wang P. The parasympathetic nervous system in the quest for stroke therapeutics. J Cereb Blood Flow Metab 2011;31:1187-1195.

158. Yu Z, Weller RA, Sandidge $K$, Weller EB. Vagus nerve stimulation: can it be used in adolescents or children with treatment-resistant depression? Curr Psychiatry Rep 2008;10:116122.

159. Martin A, Domercq M, Matute $C$. Inflammation in stroke: the role of cholinergic, purinergic and glutamatergic signaling. Ther Adv Neurol Disord 2018;11:1756286418774267.

160. Miller AH, Maletic $V$, Raison CL. Inflammation and its dis- contents: the role of cytokines in the pathophysiology of major depression. Biol Psychiatry 2009;65:732-741.

161. Follesa P, Biggio F, Gorini G, Caria S, Talani G, Dazzi L, et al. Vagus nerve stimulation increases norepinephrine concentration and the gene expression of BDNF and bFGF in the rat brain. Brain Res 2007;1179:28-34.

162. Kaniusas $E_{1}$ Kampusch $S$, Tittgemeyer M, Panetsos F, Gines RF, Papa $M$, et al. Current directions in the auricular vagus nerve stimulation II: an engineering perspective. Front $\mathrm{Neu}$ rosci 2019;13:772.

163. Dawson J, Pierce D, Dixit A, Kimberley TJ, Robertson M, Tarver $B$, et al. Safety, feasibility, and efficacy of vagus nerve stimulation paired with upper-limb rehabilitation after ischemic stroke. Stroke 2016;47:143-150.

164. Khodaparast N, Hays SA, Sloan AM, Hulsey DR, Ruiz A, Pantoja $M$, et al. Vagus nerve stimulation during rehabilitative training improves forelimb strength following ischemic stroke. Neurobiol Dis 2013;60:80-88.

165. Khodaparast N, Kilgard MP, Casavant R, Ruiz A, Qureshi I, Ganzer PD, et al. Vagus nerve stimulation during rehabilitative training improves forelimb recovery after chronic ischemic stroke in rats. Neurorehabil Neural Repair 2016;30:676684.

166. Meyers EC, Solorzano BR, James J, Ganzer PD, Lai ES, Rennaker RL 2nd, et al. Vagus nerve stimulation enhances stable plasticity and generalization of stroke recovery. Stroke 2018;49:710-717.

167. Ay I, Nasser R, Simon B, Ay H. Transcutaneous cervical vagus nerve stimulation ameliorates acute ischemic injury in rats. Brain Stimul 2016;9:166-173. 